\title{
Analysis of critical molecules and signaling pathways in osteoarthritis and rheumatoid arthritis
}

\author{
FENG XUE $^{1 *}$, CHANGQING ZHANG $^{2 *}$, ZHIMIN HE $^{1}$, LIANG DING $^{1}$ and HAIJUN XIAO ${ }^{1}$ \\ ${ }^{1}$ Department of Orthopaedics, Fengxian Central Hospital, Shanghai 201400; ${ }^{2}$ Department of Orthopaedics, \\ The Six People's Hospital Affiliated to Shanghai Jiao Tong University, Shanghai 200233, P.R. China
}

Received July 1, 2012; Accepted October 22, 2012

DOI: $10.3892 / \mathrm{mmr} .2012 .1224$

\begin{abstract}
Osteoarthritis (OA) and rheumatoid arthritis (RA) are the most prevalent forms of arthritis in the elderly. This study aimed to explore the molecular mechanisms of these diseases and identify underlying therapeutic targets. Using GSE1919 microarray data sets downloaded from the Gene Expression Omnibus database, we screened differentially expressed genes (DEGs) in OA and RA cells. The underlying molecular mechanisms of these crucial genes were investigated by Kyoto Encyclopedia of Genes and Genomes pathway enrichment analysis. Small molecule expression and SNP analysis were also conducted by searching CMap and dbSNP databases. More than 320 genes changed in the arthritic cells and there were only 196 DEGs between OA and RA. OA and RA activated the classic mitogen-activated protein kinase signaling pathway, insulin signaling pathway, antigen processing and presentation and intestinal immune network for IgA production. Graftversus-host disease and autoimmune thyroid disease-related pathways were also activated in OA and RA. Parthenolide and alsterpaullone may be treatments for OA and RA and insulinlike growth factor 1, collagen $\alpha 2$ (I) chain and special AT-rich sequence-binding protein 2 may be critical SNP molecules in arthritis. Our findings shed new light on the common molecular mechanisms of OA and RA and may provide theoretical support for further clinical therapeutic studies.
\end{abstract}

\section{Introduction}

Osteoarthritis (OA) is a group of states associated with defective articular cartilage and changes in the underlying bone. $\mathrm{OA}$ is divided into erosive or non-erosive. Erosive OA is more abrupt and commonly exhibits subchondral bone erosions (1). Pathological changes in articular cartilage and subchondral

Correspondence to: Dr Haijun Xiao, Department of Orthopaedics, Fengxian Central Hospital, Fengxian District, 9588 Nanfeng Road, Shanghai 201400, P.R. China

E-mail: xiaohaijun7@gmail.com

*Contributed equally

Key words: osteoarthritis, rheumatoid arthritis, signaling pathways bone result from chondrocyte imbalance in the extracellular matrix. Although numerous studies have reported probable chemical or mechanical causes of cartilage destruction $(2,3)$, this area of research requires more detailed investigation.

Rheumatoid arthritis (RA) is a complex, chronic multisystemic autoimmune disease, which affects the synovial membranes of multiple joints, cartilage and bone as well as bursa and tendon sheaths (4). RA is a prevalent chronic inflammatory joint disease affecting $0.5-1 \%$ of the world's population (5). RA leads to severe morbidity and disability if incorrectly treated, imposing a substantial economic burden on the affected individuals and society. The inflammatory process associated with RA is primarily observed in the synovial tissue. Synovial hyperplasia results from synovial outgrowths or synovial villi, comprised of macrophages, synovial lining cells, lymphocytes and blood vessels (6). Joint destruction occurs when the synovial pannus produces enzymes resulting in cartilage penetration, cartilage damage and joint erosion (7).

Although RA and OA share similar symptoms, it has been demonstrated that RA follows an alternative inflammatory pathway of pathogenesis to OA. Diagnosis and assessment of RA and OA is largely based on semi-quantitative methods of diagnosis, including symptoms, joint damage and physical function (8). At present, no cure exists for RA and OA and the management of these diseases depends upon early detection and aggressive treatment. Therefore, it is increasingly important to explore the molecular mechanisms of these diseases and analyze the associated signaling pathways, in order to uncover an effective therapeutic approach. In this study, we analyzed gene expression profiles of OA and RA cells to determine differentially expressed genes (DEGs) in the two forms of arthritis. Furthermore, through comparison we determined changed metabolic and non-metabolic pathways, small bioactive molecules and SNP corresponding genes associated with RA and OA.

\section{Materials and methods}

Gene expression profiles of synovial tissue samples from $R A$ and $O A$ patients and normal donors. The transcription profile of GSE1919 was obtained from the National Center for Biotechnology Information Gene Expression Omnibus database (http://www.ncbi.nlm.nih.gov/geo/) and was based on the Affymetrix Human Genome U95A Array (Santa Clara, CA, USA). A total of 15 chips were used in this study, including 
5 OA tissue chips, 5 RA tissue chips and 5 normal donor (ND) tissue chips (9). The study was approved by the Ethics Committee of the Charité Universitätsmedizin Berlin and has been performed in accordance with the ethical standards laid down in the 1964 Declaration of Helsinki. All patients gave their informed consent prior to their inclusion in the study.

Analysis of DEGs. Raw data were normalized using the robust multichip average method (10) with the default settings implemented in the $\mathrm{R}$ affy package (version 2.13.0). The Limma (linear models for microarray data) method was used to identify DEGs (11). The original expression datasets from all conditions were extracted into expression estimates and used to construct the linear model. Significance of gene expression differences between OA, RA and ND cells were tested by classical t-test and P-values were adjusted for multiple comparisons using the false discovery rate (FDR) of Benjamini and Hochberg (12). FDR-corrected $\mathrm{P}<0.05$ was considered to indicate a statistically significant difference.

Pathway analysis. Kyoto Encyclopedia of Genes and Genomes (KEGG) pathway database is a collection of manually drawn pathway maps of the molecular interaction and reaction networks. A total of 130 pathways, involving 2,287 genes, were collected from KEGG (updated 2011/06). The Database for Annotation, Visualization and Integrated Discovery (DAVID), a highthroughput and integrated data-mining environment, analyzed gene lists derived from high-throughput genomic experiments. The DAVID $(13,14)$ was used to identify over-represented pathways based on the hypergeometric distribution. Pathways with $\mathrm{P}<0.05$ and count $>2$ were considered to be significant.

Small molecule expression analysis. CMap (the connectivity map) is a collection of gene expression profiles from cultured human cells treated with bioactive small molecules. The database contains 6,100 bioactive small molecule-interfering tests and 7,056 corresponding gene expression profiles (15). DEGs were analyzed through the CMap database to identify small bio-active molecules which resulted in similar or adverse gene expression. Probes of DEGs were converted to the accession number of GeneBank and then probe numbers for use in the CMap.

$R A$ - and OA-related SNP analysis. RA- and OA- related SNPs were obtained following a dbSNP database search (http://www. ncbi.nlm.nih.gov/projects/SNP/) using the keywords 'osteoarthritis' and 'osteoporosis'. SNPs of RA and OA, which were suitable for probing, were acquired through the comparison of their corresponding genes with DEGs under pathological conditions.

\section{Results}

Recognition of DEGs in different samples. Analysis of GSE1919 using the Limma method identified a total of 460 DEGs in OA tissues compared with normal tissues. In RAt issues 1,148 DEGs were identified in comparison with ND $(\mathrm{P}<0.05$; Fig. 1). Only 196 DEGs were identified when we compared the gene expression in OA tissues with that of RA. These results indicate similarities in the molecular mechanisms of OA and RA.

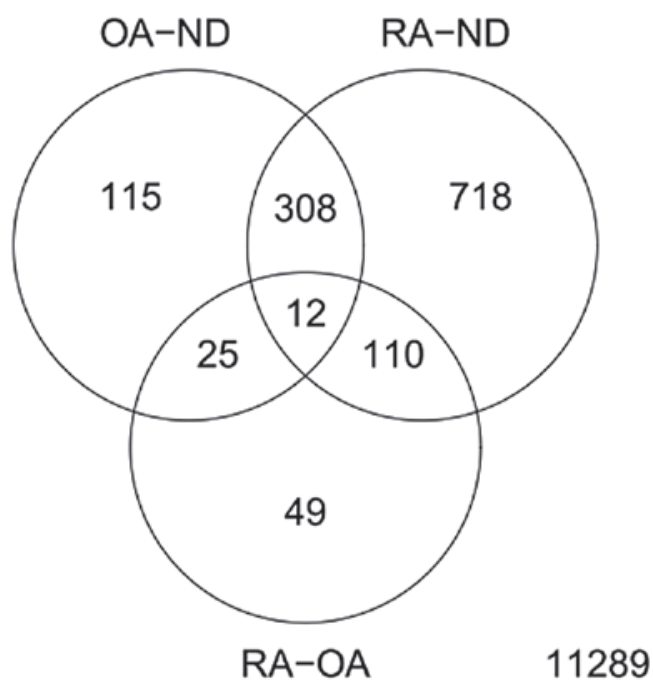

Figure 1. Venn diagram indicates the number of overlapping genes differentially expressed between normal and arthritic tissues. Of the 11,289 screened genes from micorarray, 1337 were differentially expressed between normal and arthritic tissues, including 460 genes between OA and ND, 1148 genes between RA and ND, and 196 genes between RA and OA. -, comparison between two groups. OA, osteoarthritis; RA, rheumatoid arthritis; ND, normal donor.

Analysis of pathways induced by arthritis. In arthritic tissues, gene expression profiles were significantly changed compared with the normal tissues. Therefore, DEGs were adopted to perform KEGG sub-pathway enrichment analysis. Significantly changed pathways with $\geq 2$ genes included and $\mathrm{P}<0.05$ in arthritis were obtained. It was demonstrated that the majority of pathways were involved in metabolic and nonmetabolic processes, indicative of a large number of changes in arthritic tissues compared with normal tissues (Table I). These results are likely to be important for drug discovery for the treatment of arthritis.

Analysis of small molecules resulting in RA and OA. DEGs were first divided into upregulated and downregulated genes and then enriched with significantly changed genes obtained from treatment of small molecules from the CMap database. Targeted molecules observed to induce similar effects to arthritis were selected and the 20 targeted molecules with the lowest P-values were enumerated (Table II). Parthenolide and alsterpaullone were identified in OA and RA tissue analysis and are highly relevent molecules.

Analysis of RA- and OA-related SNPs. A total of 10 SNPs were obtained from the dbSNP database with the keyword 'osteoarthritis' and 15 were obtained with the keyword 'osteoporosis'. Following comparison of these acquired SNP corresponding genes with DEGs, we revealed that no arthritis-related SNP corresponding genes were the same as the previously identified DEGs and three osteoporosis-related SNP corresponding genes were identified in the DEGs (Table III).

\section{Discussion}

During the development of OA and RA, significant changes in gene expression occur. The present study demonstrated that more than 320 genes changed in OA and RA. Study of these 
Table I. Difference in signaling pathways between normal and arthritic tissues.

Arthritic

tissue

Term

P-value

OA-ND hsa04612:Antigen processing and presentation

7.07E-04

hsa05322:Systemic lupus

erythematosus

7.72E-04

hsa04910:Insulin signaling

pathway

0.001034

hsa05332:Graft-versus-host disease

0.002426

hsa03320:PPAR signaling pathway

0.003083

hsa04940:Type I diabetes mellitus

0.003567

hsa05416:Viral myocarditis

hsa05330:Allograft rejection

0.00369

0.008703

hsa04640:Hematopoietic cell lineage

0.011659

hsa04010:MAPK signaling pathway

hsa05310:Asthma

0.014961

hsa04514:Cell adhesion molecules

hsa04142:Lysosome

0.019068

0.019897

0.024332

hsa04672:Intestinal immune network

for $\operatorname{Ig}$ A production

0.030296

hsa04920:Adipocytokine signaling

pathway

hsa05320:Autoimmune thyroid disease

RA-OA hsa04060:Cytokine-cytokine receptor interaction

hsa05340:Primary immunodeficiency

hsa04062:Chemokine signaling pathway

hsa04630:Jak-STAT signaling pathway

hsa04650:Natural killer cell-mediated

cytotoxicity

hsa04660:T cell receptor signaling

pathway

hsa04672:Intestinal immune network

for $\operatorname{Ig} \mathrm{A}$ production

hsa02010:ABC transporters

hsa04510:Focal adhesion

hsa04640:Hematopoietic cell lineage

RA-ND hsa04666:Fc $\gamma$ R-mediated

phagocytosis

hsa04612:Antigen processing and

presentation

0.032485

0.035251

$2.55 \mathrm{E}-06$

$1.52 \mathrm{E}-05$

0.002685

0.003031

0.004972

0.007133

0.00735

0.032296

0.040002

0.04746

7.49E-10

$2.56 \mathrm{E}-07$

hsa04062:Chemokine signaling pathway

hsa04672:Intestinal immune network for

IgA production

hsa 05330:Allograft rejection

hsa04514:Cell adhesion molecules

hsa04940:Type I diabetes mellitus

hsa05416:Viral myocarditis

hsa05332:Graft-versus-host disease

hsa05322:Systemic lupus erythematosus

hsa04650:Natural killer cell-mediated

cytotoxicity

hsa05310:Asthma
Table I. Continued.

Arthritic

tissue

Term

P-value

hsa04662:B cell receptor signaling

pathway

3.72E-05

hsa04660:T cell receptor signaling

pathway

3.87E-05

hsa05340:Primary immunodeficiency

hsa04670:Leukocyte transendothelial

migration

4.24E-05

hsa04640:Hematopoietic cell lineage

hsa04620:Toll-like receptor signaling

pathway

$1.71 \mathrm{E}-04$

$2.68 \mathrm{E}-04$

hsa05320:Autoimmune thyroid disease

hsa04664:Fce RI signaling

pathway

3.16E-04

$5.90 \mathrm{E}-04$

hsa04010:MAPK signaling pathway

6.59E-04

hsa04060:Cytokine-cytokine receptor

interaction

0.001477

hsa04520:Adherens junction

0.001906

hsa04142:Lysosome

0.004307

0.005237

hsa04722:Neurotrophin signaling

pathway

0.019966

hsa05210:Colorectal cancer

0.022059

hsa04512:ECM-receptor interaction

0.022059

hsa04910:Insulin signaling pathway

0.02415

hsa04510:Focal adhesion

0.031928

hsa05221:Acute myeloid leukemia

0.039772

hsa04810:Regulation of actin

cytoskeleton

0.04152

hsa05120:Epithelial cell signaling in

Helicobacter pylori infection

0.048088

OA, osteoarthritis; RA, rheumatoid arthritis; ND, normal donor.

common DEGs may help identify potential broad-spectrum anti-arthritis drugs. Furthermore, only 196 DEGs were identified between OA and RA, including interleukin 3 receptor $\alpha$ $(I L 3 R A)$, transforming growth factor $\beta$ receptor III and $C R Y A B$, indicating that the two diseases are correlated and drugs that simultaneously treat these diseases may exist.

Cluster analysis of DEGs demonstrated several common pathways associated with these diseases, including the classic mitogen-activated protein kinase (MAPK) signaling pathway and the insulin signaling pathway. The signaling pathways leading to MAPK activation have been linked to various catabolic responses in diseases, including arthritis $(16,17)$. Two immune pathways, antigen processing and presentation and intestinal immune network for IgA production, were also activated in the arthritic tissues, indicating that the immune response is involved in these diseases. Inflammation and cytokines play significant roles in RA and in certain cases of OA (17). Furthermore, changes of several cell adhesion molecules, including integrin $\beta 2$ (ITGB2) (18) and protein tyrosine phosphatase receptor 
Table II. Intersection of gene expressions between small bioactive molecules and the differentially expressed genes of arthritis.

\begin{tabular}{|c|c|c|}
\hline Arthritic tissue & CMap name & P-value \\
\hline \multirow[t]{20}{*}{ OA vs. ND } & Doxorubicin & 0 \\
\hline & $\mathrm{H}-7$ & 0 \\
\hline & Alsterpaullone & 0 \\
\hline & GW-8510 & 0 \\
\hline & Anisomycin & 0 \\
\hline & Thapsigargin & 0 \\
\hline & MG-262 & 0 \\
\hline & Parthenolide & 0 \\
\hline & Withaferin A & 0 \\
\hline & Cephaeline & 0 \\
\hline & 15-delta prostaglandin $\mathrm{J} 2$ & 0 \\
\hline & Mitoxantrone & 0.00002 \\
\hline & Valinomycin & 0.00006 \\
\hline & Disulfiram & 0.00016 \\
\hline & Lomustine & 0.00024 \\
\hline & Terfenadine & 0.00026 \\
\hline & Lanatoside C & 0.00026 \\
\hline & Gossypol & 0.00052 \\
\hline & 5224221 & 0.00056 \\
\hline & 5194442 & 0.00062 \\
\hline \multirow[t]{20}{*}{ RA vs. ND } & Thapsigargin & 0 \\
\hline & Parthenolide & 0 \\
\hline & Niclosamide & 0 \\
\hline & Alsterpaullone & 0.00002 \\
\hline & Helveticoside & 0.00016 \\
\hline & Valinomycin & 0.0003 \\
\hline & Fluticasone & 0.00097 \\
\hline & 5194442 & 0.00105 \\
\hline & Cephaeline & 0.00134 \\
\hline & Diphenylpyraline & 0.00179 \\
\hline & Tiapride & 0.00192 \\
\hline & Methylergometrine & 0.00219 \\
\hline & Metixene & 0.00292 \\
\hline & Methyldopate & 0.00294 \\
\hline & Lanatoside C & 0.00326 \\
\hline & CP-320650-01 & 0.00329 \\
\hline & Enoxacin & 0.00394 \\
\hline & Procainamide & 0.004 \\
\hline & CP-690334-01 & 0.00445 \\
\hline & Tranylcypromine & 0.00449 \\
\hline
\end{tabular}

OA, osteoarthritis; RA, rheumatoid arthritis; ND, normal donor.

type c (PTPRC) (19) and lysosome-related molecules, including phospholipase A2 group XV (PLA2G15) (20) and adaptorrelated protein complex $1 \beta(A P 1 B 1)(21)$ in OA and RA cells demonstrated that the two diseases altered their microenvironment and removed the exogenous substances by the lysosome. In addition, graft-versus-host (22) and autoimmune thyroid
Table III. Corresponding differently expressed genes of disease-related SNPs.

\begin{tabular}{lll}
\hline Arthritic tissue & Gene & \multicolumn{1}{c}{ SNP ID } \\
\hline OA-ND & IGF1 & 121912430 \\
& COL1A2 & 72658152 \\
RA-ND & IGF1 & $1 \mathrm{E}+08$ \\
& SATB2 & $1 \mathrm{E}+08$ \\
\hline
\end{tabular}

OA, osteoarthritis; RA, rheumatoid arthritis; ND, normal donor. SNP ID represents the identification number from the dbSNP database. SNP, single nucleotide polymorphism.

disease-related pathways were also activated in OA and RA (23). Further investigation of these pathways is likely to be shed light the network of signal pathways under OA and RA. More studies on the DEGs between OA and RA may provide useful information to differentiate the molecular mechanisms associated with $\mathrm{OA}$ and RA.

Based on the DEGs and data from the CMap database, we acquired a series of small molecules. Two of these small molecules, parthenolide and alsterpaullone, demonstrated significant similarity in $\mathrm{OA}$ and RA tissues $(\mathrm{P}<0.05)$ and required additional analysis to determine their suitability as broad spectrum anti-arthritis drugs.

Parthenolide is a sesquiterpene lactone of the germacranolide class which occurs naturally in the plant feverfew (Tanacetum parthenium). Parthenolide modulates the NF- $\kappa \mathrm{B}$-mediated inflammatory responses in experimental atherosclerosis (24) and blocks lipopolysaccharide-induced osteolysis through suppression of NF- $\kappa \mathrm{B}$ activity (25). Parthenolide induces apoptosis in acute myelogenous leukemia cells, leaving normal bone marrow cells relatively unscathed (26). Pharthenolide also exhibits microtubule-interfering activity (27), anti-inflammatory and anti-hyperalgesic effects (28) and activity against the parasite Leishmania amazonensis (29). Since numerous cases of OA and RA result from the interruption of immune responses, including inflammation, it is probable that parthenolide is a suitable therapeutic for these ailments.

Alsterpaullone is a potent, ATP-competitive inhibitor of the cell cycle regulating cyclin-dependent kinases CDK1/cyclin B $\left(\mathrm{IC}_{50}=0.035 \mu \mathrm{M}\right)$ and an inhibitor of GSK-3 $\beta$ and the neuronal CDK5/p25 (30). In addition, alsterpaullone induces apoptosis by activation of caspase- 8 and -9 followed by disruption of mitochondrial potential (31).

Through analysis of RA- and OA-related SNPs, we identified that three osteoporosis-related SNP corresponding genes (IGF1, COL1A2 and SATB2) were differentially expressed. Insulin-like growth factor 1 (IGF-1), also called somatomedin C, is a protein encoded by the IGF1 gene in humans (32,33). IGF-1 is expressed and produced by chondrocytes and is one of the anabolic growth factors associated with cartilage (34) and thus is involved in arthritis. Collagen $\alpha 2(\mathrm{I})$ chain is a protein encoded by the COL1A2 gene in humans $(35,36)$. Mutations in this gene are associated with osteogenesis imperfecta, Ehlers-Danlos syndrome, idiopathic osteoporosis and atypical Marfan syndrome. Special AT-rich sequence-binding protein 2 
(SATB2), also known as DNA-binding protein SATB2, is a human protein encoded by the SATB2 gene (37). SATB2 has been identified to be disrupted in two unrelated cases with de novo apparently balanced chromosome translocations associated with cleft palate and Pierre Robin Sequence (38). The present study also demonstrated that these DEGs were often mutated under arthritis and thereby more studies should focus on their roles in OA and RA.

The present findings shed new light on the molecular mechanisms of OA and RA. Results revealed more than 320 DEGs in both diseases which may be involved in OA and RA development via MAPK and insulin signaling pathways, antigen processing and presentation, intestinal immune network, graftversus-host disease and autoimmune thyroid disease-related pathways. Notably, parthenolide and alsterpaullone were identified as important small molecules involved in the induction of anti-inflammatory and apoptosis-related gene expression and thus we suggest that these molecules may be suitable anti-arthritis drugs for OA and RA. Furthermore, mutations of $I G F 1, C O L 1 A 2$ and SATB2 genes were critical for the pathogenesis of OA and RA. However, there are specific limitations in our study. The pathway enrichment was only based on the connection between genes and therefore genes without strong neighbors are likely to be excluded from the analysis (13). In addition, further experimental analysis is required to confirm the conclusions of the present study.

\section{References}

1. Roach HI: The complex pathology of osteoarthritis: even mitochondria are involved. Arthritis Rheum 58: 2217-2218, 2008.

2. Berenbaum F: New horizons and perspectives in the treatment of osteoarthritis. Arthritis Res Ther 10 (Suppl 2): S1, 2008.

3. Dieppe P: Osteoarthritis of the knee in primary care. BMJ 336: 105-106, 2008

4. Gay S, Gay RE and Koopman WJ: Molecular and cellular mechanisms of joint destruction in rheumatoid arthritis: two cellular mechanisms explain joint destruction? Ann Rheum Dis 52 (Suppl 1): S39-S47, 1993.

5. Kvien TK: Epidemiology and burden of illness of rheumatoid arthritis. Pharmacoeconomics 22: 1-12, 2004.

6. Beasley J: Osteoarthritis and rheumatoid arthritis: conservative therapeutic management. J Hand Ther 25: 163-171, 2012.

7. Scott DL and Symmons DP: The role of specialists in managing established rheumatoid arthritis. Rheumatology (Oxford) 47: 237-238, 2008.

8. Biswas S, Manikandan J and Pushparaj PN: Decoding the differential biomarkers of Rheumatoid arthritis and Osteoarthritis: A functional genomics paradigm to design disease specific therapeutics. Bioinformation 6: 153-157, 2011.

9. Ungethuem U, Haeupl T, Witt H, et al: Molecular signatures and new candidates to target the pathogenesis of rheumatoid arthritis. Physiol Genomics 42A: 267-282, 2010.

10. Irizarry RA, Hobbs B, Collin F, et al: Exploration, normalization and summaries of high density oligonucleotide array probe level data. Biostatistics 4: 249-264, 2003 .

11. Smyth GK: Linear models and empirical bayes methods for assessing differential expression in microarray experiments. Stat Appl Genet Mol Biol 3: 3, 2004.

12. Benjamini Y and Hochberg Y: Controlling the false discovery rate: a practical and powerful approach to multiple testing. J R Stat Soc Series B Stat Methodol 57: 289-300, 1995.

13. Huang DW, Sherman BT and Lempicki RA: Systematic and integrative analysis of large gene lists using DAVID bioinformatics resources. Nat Protoc 4: 44-57, 2009.

14. Huang da W, Sherman BT and Lempicki RA: Bioinformatics enrichment tools: paths toward the comprehensive functional analysis of large gene lists. Nucleic Acids Res 37: 1-13, 2009.

15. Lamb J, Crawford ED, Peck D, et al: The connectivity map: using gene-expression signatures to connect small molecules, genes and disease. Science 313: 1929-1935, 2006.
16. Sondergaard BC, Schultz N, Madsen SH, Bay-Jensen AC, Kassem M and Karsdal MA: MAPKs are essential upstream signaling pathways in proteolytic cartilage degradation-divergence in pathways leading to aggrecanase and MMP-mediated articular cartilage degradation. Osteoarthritis Cartilage 18: 279-288, 2010.

17. Lawrence MC, Jivan A, Shao C, et al: The roles of MAPKs in disease. Cell Res 18: 436-442, 2008.

18. Kotovuori A, Pessa-Morikawa T, Kotovuori P, Nortamo P and Gahmberg CG: ICAM-2 and a peptide from its binding domain are efficient activators of leukocyte adhesion and integrin affinity. J Immunol 162: 6613-6620, 1999.

19. Kaplan R, Morse B, Huebner K, et al: Cloning of three human tyrosine phosphatases reveals a multigene family of receptorlinked protein-tyrosine-phosphatases expressed in brain. Proc Natl Acad Sci U S A 87: 7000-7004, 1990.

20. Wang A and Dennis EA: Mammalian lysophospholipases. Biochim Biophys Acta 1439: 1-16, 1999

21. Peyrard M, Fransson I, Xie YG, et al: Characterization of a new member of the human beta-adaptin gene family from chromosome $22 \mathrm{q} 12$, a candidate meningioma gene. Hum Mol Genet 3: 1393-1399, 1994.

22. Ferrara JL, Levine JE, Reddy P and Holler E: Graft-versus-host disease. Lancet 373: 1550-1561, 2009.

23. Weetman AP and McGregor AM: Autoimmune thyroid disease: further developments in our understanding. Endocr Rev 15: 788-830, 1994.

24. López-Franco O, Hernández-Vargas P, Ortiz-Muñoz G, et al: Parthenolide modulates the NF-kappaB-mediated inflammatory responses in experimental atherosclerosis. Arterioscler Thromb Vasc Biol 26: 1864-1870, 2006.

25. Yip KH, Zheng MH, Feng HT, Steer JH, Joyce DA and Xu J: Sesquiterpene lactone parthenolide blocks lipopolysaccharideinduced osteolysis through the suppression of NF-kappaB activity. J Bone Miner Res 19: 1905-1916, 2004.

26. Guzman ML, Rossi RM, Karnischky L, et al: The sesquiterpene lactone parthenolide induces apoptosis of human acute myelogenous leukemia stem and progenitor cells. Blood 105: 4163-4169, 2005

27. Miglietta A, Bozzo F, Gabriel L and Bocca C: Microtubuleinterfering activity of parthenolide. Chem Biol Interact 149: $165-173,2004$

28. Feltenstein MW, Schuhly W, Warnick JE, Fischer NH and Sufka KJ: Anti-inflammatory and anti-hyperalgesic effects of sesquiterpene lactones from Magnolia and Bear's foot. Pharmacol Biochem Behav 79: 299-302, 2004.

29. Tiuman TS, Ueda-Nakamura T, Garcia Cortez DA, et al: Antileishmanial activity of parthenolide, a sesquiterpene lactone isolated from Tanacetum parthenium. Antimicrob Agents Chemother 49: 176-182, 2005.

30. Leost M, Schultz C, Link A, et al: Paullones are potent inhibitors of glycogen synthase kinase-3beta and cyclin-dependent kinase 5/p25. Eur J Biochem 267: 5983-5994, 2000.

31. Lahusen T, De Siervi A, Kunick C and Senderowicz AM Alsterpaullone, a novel cyclin-dependent kinase inhibitor, induces apoptosis by activation of caspase-9 due to perturbation in mitochondrial membrane potential. Mol Carcinog 36: 183-194, 2003.

32. Hoppener JW, de Pagter-Holthuizen P, Geurts van Kessel AH, et al: The human gene encoding insulin-like growth factor I is located on chromosome 12. Hum Genet 69: 157-160, 1985.

33. Jansen M, van Schaik FM, Ricker AT, et al: Sequence of cDNA encoding human insulin-like growth factor I precursor. Nature 306: 609-611, 1983.

34. Studer RK: Nitric oxide decreases IGF-1 receptor function in vitro; glutathione depletion enhances this effect in vivo. Osteoarthritis Cartilage 12: 863-869, 2004.

35. Retief E, Parker MI and Retief AE: Regional chromosome mapping of human collagen genes alpha 2(I) and alpha 1(I) (COLIA2 and COLIA1). Hum Genet 69: 304-308, 1985.

36. Wenstrup RJ, Cohn DH, Cohen T and Byers PH: Arginine for glycine substitution in the triple-helical domain of the products of one alpha 2(I) collagen allele (COL1A2) produces the osteogenesis imperfecta type IV phenotype. J Biol Chem 263: 7734-7740, 1988.

37. Kikuno R, Nagase T, Ishikawa K, et al: Prediction of the coding sequences of unidentified human genes. XIV. The complete sequences of 100 new cDNA clones from brain which code for large proteins in vitro. DNA Res 6: 197-205, 1999.

38. FitzPatrick DR, Carr IM, McLaren L, et al: Identification of SATB2 as the cleft palate gene on 2q32-q33. Hum Mol Genet 12: 2491-2501, 2003. 